\title{
双环[3.3.1]壬烷-2,6,9-三酮的合成
}

\author{
任晓莉凌亦飞罗 军* \\ (南京理工大学化工学院 南京 210094)
}

\begin{abstract}
摘要 研究了新化合物双环[3.3.1]壬烷-2,6,9-三酮以 1,3-环己二酮为起始原料的三条合成路线, 并对其反应机理进行了 初步推测. 第一条路线以 1,3-环己二酮与丙烯醛发生 Michael 加成、分子内 aldol 缩合以及氧化三步得到双环 [3.3.1]壬 烷-2,6,9-三酮，总产率为 $43 \%$. 第二条路线为 1,3 -环己二酮先与吗啉反应生成烯胺，再与丙烯酸乙酯进行环合，以 “一 锅煮” 法得到目标产物, 产率为 $20 \%$. 第三条路线为 1,3-环己二酮先与丙烯酸乙酯经过 Michael 加成, 再进行酸催化分 子内 C-酰化得到目标产物, 总产率为 $83 \%$, 该路线具有操作简单、条件温和、产率高及环境友好等优点. 关键词 1,3-环己二酮; 双环[3.3.1]壬烷-2,6,9-三酮; 丙烯醛; 丙烯酸乙酯; 环化
\end{abstract}

\section{Synthesis of Bicyclo[3.3.1]nonane-2,6,9-trione}

\author{
Ren, Xiaoli Ling, Yifei Luo, Jun* \\ (School of Chemical Engineering, Nanjing University of Science \& Technology, Nanjing 210094)
}

\begin{abstract}
A novel compound bicyclo[3.3.1]nonane-2,6,9-trione was synthesized from cyclohexane-1,3-dione through three routes and some reaction mechanisms were proposed. The first route afforded bicyclo[3.3.1]nonane-2,6,9-trione in a total yield of $43 \%$ via Michael addition with cyclohexane-1,3-dione and acrolein, intermolecular Aldol condensation and oxidation. The second route gave target product in $20 \%$ yield via a one-pot process including formation of enamine with morpholine and cyclization with acrylic ethyl ester. The third route also used cyclohexane-1,3-dione and acrylic ethyl ester as start materials and resulted bicyclo[3.3.1]nonane-2,6,9-trione with a high total yield of $83 \%$ via Michael addition and intermolecular C-acylation. This process has the advantages of simple operation, mild reaction conditions, high yield and environmental friendship.

Keywords cyclohexane-1,3-dione; bicyclo[3.3.1]nonane-2,6,9-trione; acrolein; acrylic ethyl ester; cyclization
\end{abstract}

双环[3.3.1]壬烷及其衍生物是一些天然产物的核心 骨架结构, 也为成环反应的研究提出了一种重要的环状 骨架 ${ }^{[1]}$. 双环[3.3.1]壬烷多酮化合物的在很多领域都有 较大用途, 如医药、高分子材料、润滑剂、高能量密度 材料、农药等 ${ }^{[2]}$. 如多环多异戊烯基间苯三酚(PPAPs) 是 一种应用日益广泛的天然产物, 这类化合物含有双环、 金刚烷和高金刚烷三酮骨架结构, 用于预防及治疗各种 肿瘤与癌症, 如对淋巴瘤、肺癌、胃癌、肌肉癌等疾病 均有一定的疗效, 代表性含双环及多环三酮骨架的天然 产物如图 1 所示 ${ }^{[3 \sim 7]}$. 近年来单环上的分子取代研究已 趋于成熟, 而双环上的分子取代, 尤其是桥头碳原子上 的取代反应，报道却较少.

Mehta 等 ${ }^{[8,9]}$ 报道了此类天然产物类似物的合成, 其 中就包含双环[3.3.1]壬烷-2,6,9-三酮骨架. 他们以取代 1,3-环已二酮为原料, 在 1,8 -二氮杂双环 [5.4.0]十一碳7-烯(DBU)催化下以 “一锅煮” 法在 2-位进行 Michael
加成和烷基化反应, 然后将酯水解生成相应的羧酸, 再 在 $\mathrm{NaOAc}-\mathrm{Ac}_{2} \mathrm{O}$ 体系中加热到 $140{ }^{\circ} \mathrm{C}$ 反应生成烯醇内 酷衍生物, 然后用 DIBAL-H 进行还原一反 aldol-aldol 串 联反应生成 9-氧代-双环[3.3.1]壬烷-2,6-二醇衍生物, 最 后用氯铬酸吡啶鎓盐( $\mathrm{PCC}$ )进行氧化得到最终的双环 [3.3.1]壬烷-2,6,9-三酮骨架. 这种方法步骤多、反应条件 苛刻、收率低. 本文通过 Michael 加成和分子内缩合等 单元反应构建了双环[3.3.1]壬烷-2,6,9-三酮骨架，研究 了三条不同的合成路线，最多两步反应即可获得目标产 物, 获得了简便、高产率的成环方法, 如 Scheme 1 所示.

\section{1 结果与讨论}

Scheme 1 所示的三条合成路线都以 1,3-环已二酮为 基本原料. Romain 等 ${ }^{[10 ~ 12]}$ 报道了 1,3-环已二酮可与丙烯 醛发生 Michael 加成生成化合物 1, 但产率仅 37\%. 在我 们研究的反应体系中发现, 生成的化合物 1 会在反应体

*E-mail: luojun@njust.edu.cn

Received September 6, 2013; revised October 22, 2013; published online October 25, 2013. 


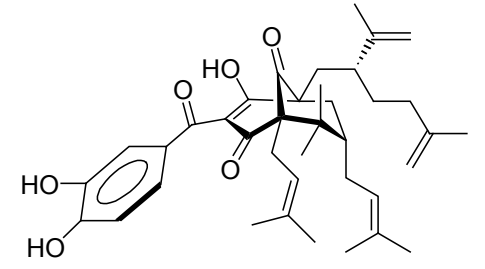

xanthochymol

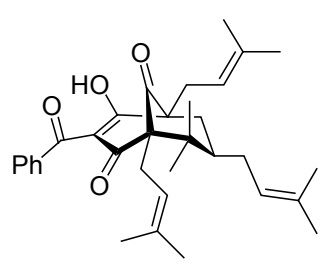

clusiamone

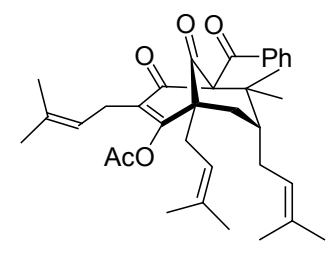

plukenetione $\mathrm{E}$

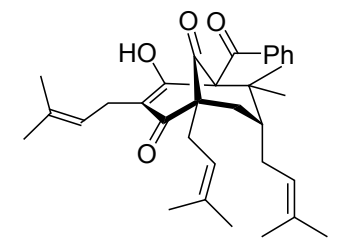

7-epi-nemorosone

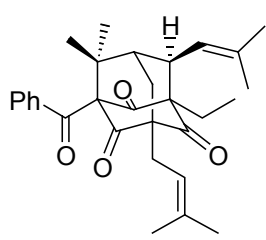

plukenetione A

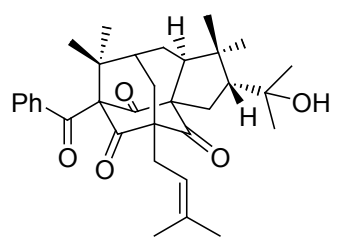

plukenetione B

图 1 含双环[3.3.1]壬烷三酮骨架的代表性天然产物

Figure 1 Selective derivatives containing bicyclo[3.3.1]nonanetrione skeleton
系中接着发生分子内 aldol 缩合生成化合物 $\mathbf{2}$, 将化合物 2 进一步氧化可以得到目标化合物 3. 还研究了路线 2 所示的 “一锅煮” 法, 本路线中 1,3-环己二酮首先与肿 胺反应生成烯胺, 再加入丙烯酸乙酯进行加成反应, 实 验证实虽有目标产物生成, 但总收率较低. 基于以上两 条路线的结果我们又设计了路线 3, 首先通过 Michael 加成生成化合物 4, 再经由酸催化发生分子内 aldol 缩合 生成化合物 3, 经过对反应条件的优化，总收率可达到 $85 \%$. 这一路线反应简单、原料易得、总产率高.

\section{1 丙烯醛法(路线 1)合成双环[3.3.1]壬烷-2,6,9-三酮}

1.1.1 1,3-环已二酮与丙烯醛的 Michael 加成-aldol 缩 合串联反应合成化合物 2

在研究催化剂对 Michael 加成-aldol 缩合串联反应 的影响时, 先后选用了活性氧化铝、哌啶、吗啉、叔丁 醇钾和碳酸钾这几种碱催化剂, 结果见表 1 . 发现在使 用吗啉和哌啶催化时产率很低, 分别只有 $25 \%$ 和 $30 \%$; 使用叔丁醇钾和碳酸钾催化 ${ }^{[13]}$ 时发现效果更差, 在反 应 $6 \mathrm{~h}$ 后原料还有大部分没有转化, 未能分离出目标产 物; 而在采用活性氧化铝为催化剂时, 发现反应较快, 原料在 $6 \mathrm{~h}$ 后转化完全(TLC 跟踪), 产率可达到 $48 \%$. 因 此后面的反应都使用活性氧化铝为催化剂. 接下来考察 了活性氧化铝用量对反应结果的影响. 发现其用量为 5 equiv.时，化合物 $\mathbf{2}$ 产率最高，再增加用量产率没有明显 变化. 因此我们确定活性氧化铝最佳用量为 5 equiv.

\subsection{2 氧化反应条件的优化}

选用了不同氧化剂对化合物 2 进行氧化, 实验结果 如表 2 所示. 发现在使用 PCC 氧化 ${ }^{[14]}$ 时, 由于反应生成

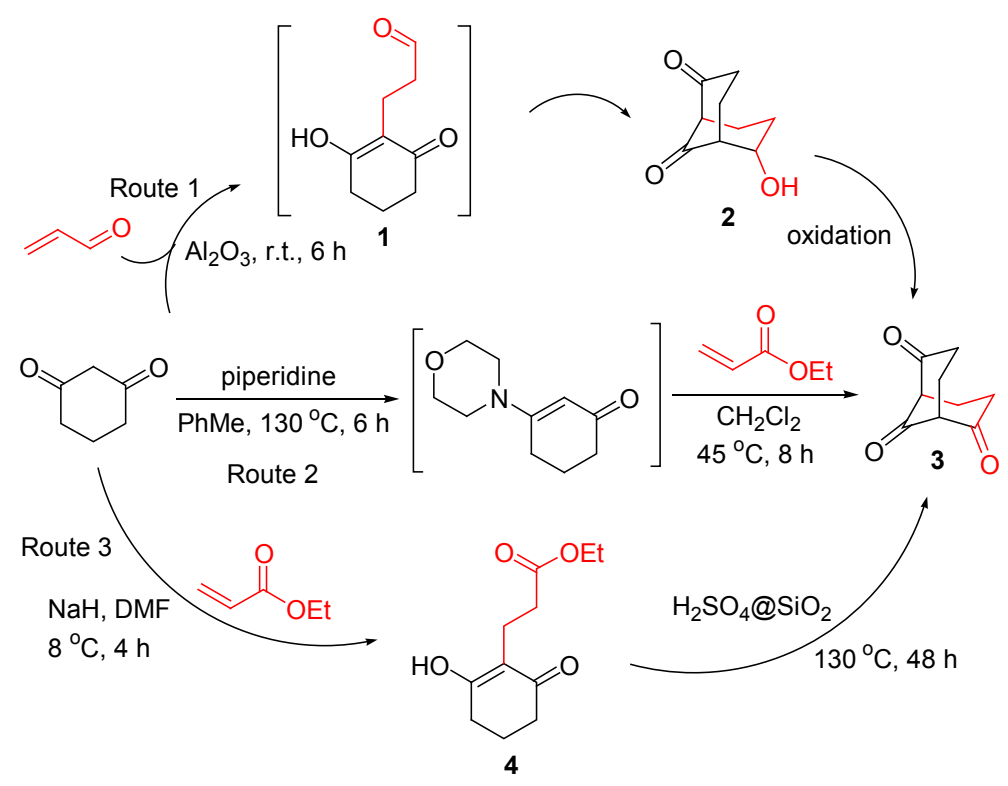

Scheme 1 
表 1 催化剂对化合物 2 产率的影响 ${ }^{a}$

Table 1 The effect of catalysts on the yield of compound 2

\begin{tabular}{clcc}
\hline Entry & Catalyst & Amount of catalyst/equiv. & Yield $^{c} / \%$ \\
\hline 1 & $\mathrm{Al}_{2} \mathrm{O}_{3}$ & 5.0 & 48 \\
2 & $\mathrm{Morpholine}^{b}$ & 0.2 & 25 \\
3 & Piperidine $^{b}$ & 0.2 & 30 \\
4 & $t$ - $\mathrm{BuK}^{b}$ & 1.5 & - \\
5 & $\mathrm{~K}_{2} \mathrm{CO}_{3}{ }^{b}$ & 1.5 & 35 \\
6 & $\mathrm{Al}_{2} \mathrm{O}_{3}$ & 4.5 & 45 \\
7 & $\mathrm{Al}_{2} \mathrm{O}_{3}$ & 4.7 & 48 \\
8 & $\mathrm{Al}_{2} \mathrm{O}_{3}$ & 5.5 & \\
\hline
\end{tabular}

${ }^{a}$ Reaction conditions: Cyclohexane-1,3-dione $1.68 \mathrm{~g}(15 \mathrm{mmol}), 25{ }^{\circ} \mathrm{C}, 6 \mathrm{~h}$, neat. ${ }^{b}$ Dichloromethane $(50 \mathrm{~mL}) .{ }^{c}$ Isolated yield.

的低价铬氧化物会对产物产生明显的吸附, 产物难以完 全分离出来，导致产率不高，只有 $55 \%$; Jones 氧化 ${ }^{[15]}$ 及 Swern 氧化 ${ }^{[16]}$ 结果也并不理想; 三氧化铬-吡啶体系氧 化时产率为 $70 \%$, 但反应剧烈、不易控制 ${ }^{[17]}$; 使用高碘 酸钠作氧化剂 ${ }^{[18]}$ 只获得了极少量产物, 高锰酸钾效果 也不佳; 而使用 $\mathrm{KHSO}_{5}$ 氧化时 ${ }^{[19]}$, 产率可达 $90 \%$, 效果 最高, 而且后处理简单, 因此确定 $\mathrm{KHSO}_{5}$ 为最佳氧化 剂. 之后考察了 $\mathrm{KHSO}_{5}$ 用量对反应结果的影响, 发现 $\mathrm{KHSO}_{5}$ 用量为 0.8 equiv. 时, 产物产率最高, 再增加氧化 剂用量产率没有明显提高. 因此 $\mathrm{KHSO}_{5}$ 最佳用量为 0.8 equiv.

表 2 氧化剂对化合物 3 产率的影响

Table 2 The effect of oxidant on the yield of compound 3

\begin{tabular}{|c|c|c|c|c|c|}
\hline \multirow{2}{*}{ Entry } & \multirow{2}{*}{ Oxidant } & \multirow{2}{*}{$\begin{array}{c}\text { Amount of } \\
\text { oxidant/equiv. }\end{array}$} & \multirow{2}{*}{$\begin{array}{l}\text { Tempera- } \\
\text { ture } /{ }^{\circ} \mathrm{C}\end{array}$} & \multicolumn{2}{|c|}{ Time/ Yieldf/ } \\
\hline & & & & $\mathrm{h}$ & $\%$ \\
\hline 1 & $\mathrm{KHSO}_{5}{ }^{a}$ & 0.80 & 70 & 7 & 90 \\
\hline 2 & $\mathrm{CrO}_{3} / \mathrm{Py}^{b}$ & $4.00 / 8.00$ & 25 & 3 & 70 \\
\hline 3 & $\mathrm{PCC}^{b}$ & 4.00 & 25 & 6 & 55 \\
\hline 4 & Jones reagent $^{c}$ & 4.00 & 0 & 5 & 30 \\
\hline 5 & $(\mathrm{COCl})_{2} / \mathrm{DMSO}^{b}$ & $1.15 / 2.24$ & -68 & 6 & 60 \\
\hline 6 & $\mathrm{KMnO}_{4}{ }^{d}$ & 2.00 & 25 & 3 & 18 \\
\hline 7 & $\mathrm{NaIO}_{4}{ }^{e}$ & 2.00 & 25 & 6 & $<5$ \\
\hline 8 & $\mathrm{KHSO}_{5}{ }^{a}$ & 0.70 & 70 & 7 & 80 \\
\hline 9 & $\mathrm{KHSO}_{5}{ }^{a}$ & 0.75 & 70 & 7 & 86 \\
\hline 10 & $\mathrm{KHSO}_{5}{ }^{a}$ & 0.85 & 70 & 7 & 90 \\
\hline
\end{tabular}

${ }^{a}$ Reaction conditions: compounds $20.8 \mathrm{~g}(4.76 \mathrm{mmol})$, ethyl acetate $(13 \mathrm{~mL})$.

${ }^{b}$ Dichloromethane $(20 \mathrm{~mL}) .{ }^{c}$ Acetone $(20 \mathrm{~mL}) .{ }^{d}$ Acetic acid $(20 \mathrm{~mL}) .{ }^{e}$ Water $(20 \mathrm{~mL}) .{ }^{f}$ Isolated yield.

\section{2 “一锅煮” 法(路线 2)合成双环[3.3.1]壬烷-2,6,9- 三酮}

\subsection{1 溶剂对反应的影响}

1,3-环已二酮在二级胺存在的条件下可以发生脱水 缩合生成烯胺 ${ }^{[20]}$. 选用吗啉与 1,3-环己二酮反应, 烯胺 形成过程中用 TLC 跟踪反应进程, 烯胺化反应结束后 加入丙烯酸乙酯, 在发生 Michael 加成反应后接着发生 分子内 C-酰化反应, 反应可以不经分离直接生成目标 产物. 考察了不同溶剂对反应结果的影响, 实验结果如
表 3 所示. 从表 3 可以看出, 虽然反应可行, 但产率较 低. 其中用二氯甲烷作为溶剂时反应可在回流状态下进 行, 产率为 $20 \%$.

表 3 溶剂对 “一锅煮” 法结果的影响 ${ }^{a}$

Table 3 The effect of solvent on the "one-pot" route for the synthesis of compound $\mathbf{3}$

\begin{tabular}{cccc}
\hline Entry & Solvent & Temperature $/{ }^{\circ} \mathrm{C}$ & Yield $^{b} \%$ \\
\hline 1 & $\mathrm{PhMe}$ & 130 & $<5$ \\
2 & $\mathrm{CH}_{3} \mathrm{CN}$ & 70 & 12 \\
3 & $\mathrm{CH}_{2} \mathrm{Cl}_{2}$ & 45 & 20 \\
\hline
\end{tabular}

${ }^{a}$ Reaction conditions: cyclohexane-1,3-dione $2.24 \mathrm{~g}(20 \mathrm{mmol})$, morpholine $5.23 \mathrm{~mL}(60 \mathrm{mmol}), 13 \mathrm{~h} .{ }^{b}$ Isolated yield.

\subsection{2 “一锅法” 反应机理的探讨}

根据实验结果, 我们推测了 “一锅法” 合成双环 [3.3.1]壬烷-2,6,9-三酮的反应机理(Scheme 2).1,3-环已 二酮与肿胺脱水缩合转变为烯胺, 其 2-位碳原子被活 化, 与丙烯酸乙酯发生 Michael 加成, 生成双性离子盐 5,5 存在互变异构体 6 , 碳负离子从环已烷环 4-位上夺 取一个氢原子(以六元环状过渡态进行)得到中间体 7 , 然后 4-位碳负离子进攻羰基碳原子，脱去乙氧基负离子 后得到双环结构 $\mathbf{8}$, 在后处理过程中进行水解得到双环 [3.3.1]壬烷-2,6,9-三酮和吗啉.

\section{3 两步法(路线 3)合成双环[3.3.1]壬烷-2,6,9-三酮}

基于以上结果, 我们调整了策略, 将 Michael 加成 反应和分子内 $\mathrm{C}$-酰化反应分两步进行. 先在碱催化下 将环已二酮与丙烯酸乙酯进行 Michael 加成反应, 发现 在优化条件下烷基化产物 4 的产率最高可达 92\%. 然后 化合物 4 再在酸催化下发生分子内 $\mathrm{C}$-酰化反应生成目 标产物 3, 产率可达到 $90 \%$. 两步反应总收率为 $83 \%$.

1.3.1 1,3-环已二酮与丙烯酸乙酯的 Michael 加成反 应

我们参考了 Mitoshi 等的条件 ${ }^{[21]}$, 选用了氢化钠作 为 1,3-环己二酮与丙烯酸乙酯的 Michael 加成反应的催 化剂, 考察了其用量对反应的影响, 结果如表 4 所示. 


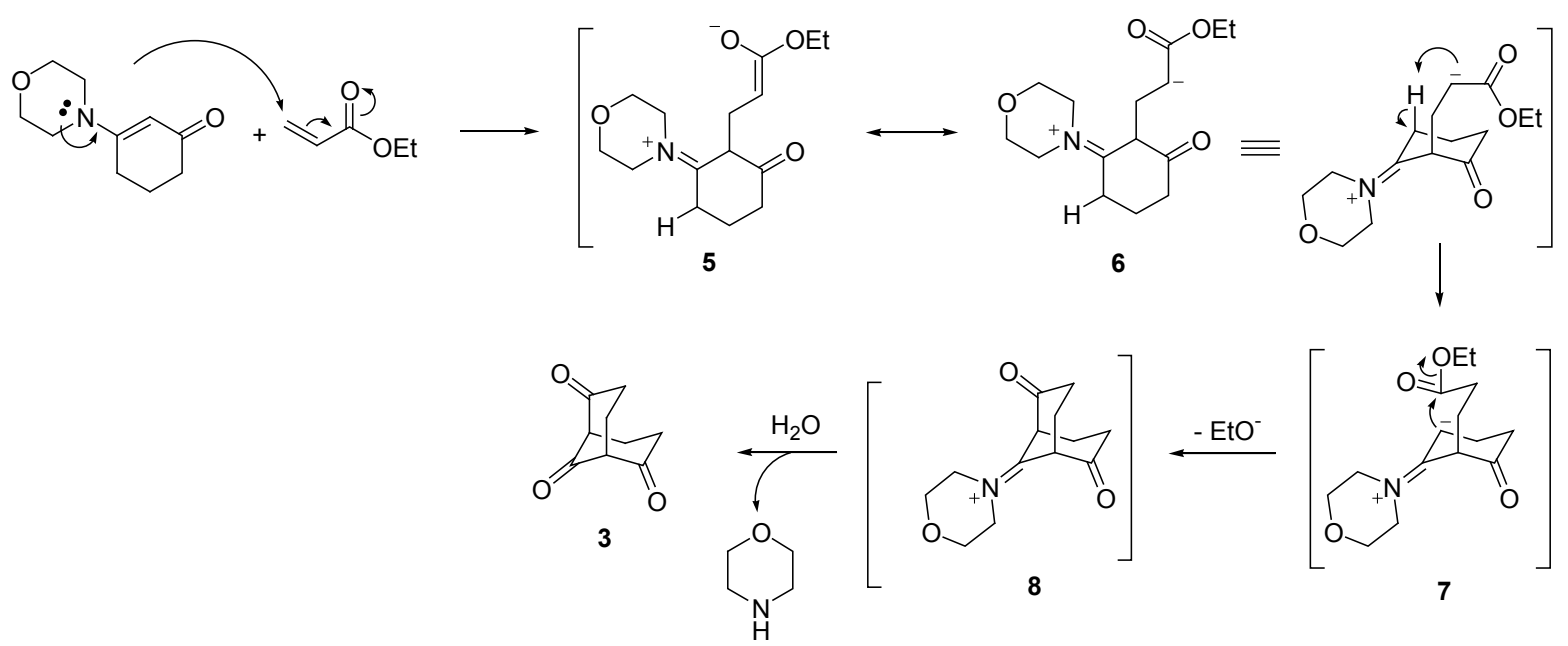

Scheme 2

发现当氢化钠的用量为 1.5 equiv. 时, 化合物 $\mathbf{4}$ 的产率最 高, 产率可达 $92 \%$.

表 $4 \mathrm{NaH}$ 用量对化合物 4 产率的影响 ${ }^{a}$

Table 4 The effect of $\mathrm{NaH}$ loading on the yield of compound 4

\begin{tabular}{ccc}
\hline Entry & NaH loading/equiv. & Yield $^{b} / \%$ \\
\hline 1 & 1.00 & 67 \\
2 & 1.25 & 80 \\
3 & 1.50 & 92 \\
3 & 1.75 & 65 \\
\hline
\end{tabular}

${ }^{a}$ Reaction conditions: Cyclohexane-1,3-dione $2.24 \mathrm{~g}(20 \mathrm{mmol})$, ethyl acrylate $2.13 \mathrm{~mL}(20 \mathrm{mmol}), 80{ }^{\circ} \mathrm{C}, 4 \mathrm{~h}, \mathrm{DMF}(8 \mathrm{~mL}) .{ }^{b}$ Isolated yield.

\subsection{2 分子内 $C$-酰化反应}

根据路线 2 的结果, 先考察了碱催化条件下的分子 内 C-酰化反应，发现使用吗啉为催化剂时几乎没有生 成产物，使用甲醇钠、哌啶、叔丁醇钾与碳酸钾时得到 相似的结果, 在使用氢化钠催化时, 发现生成的产物会 在处理过程中发生开环水解.

基于使用碱为催化剂的结果，之后改用酸作为催化 剂, 选用了多种质子酸, 实验结果如表 5 所示. 结果发 现使用酸的结果比较理想. 用浓硫酸作催化剂时, 如果 反应中不加分子篮，则酯会发生水解得到相应的羧酸; 加入分子篮可避免这种现象的发生, 但反应很慢, 经过 $48 \mathrm{~h}$ 反应也只得到 $45 \%$ 的产率. 使用多聚磷酸效果更 差, 基本上分离不出纯的产物. 选用有机酸甲基磺酸时 也不成功, 但使用对甲基苯磺酸时, 可以以 $80 \%$ 的产率 得到目标产物. 而使用硅胶负载浓硫酸时, 发现反应进 行顺利，而且最高可以得到 $90 \%$ 的产率，且原料廉价易 得，反应条件易控制，后处理及提纯方式简便. 综上考 虑, 确定硅胶负载浓硫酸为最佳酸催化剂. 在确定了最 佳催化剂之后, 研究了其用量对产率的影响, 发现当催 化剂用量为 0.4 equiv. 时产物产率最高. 因此确定催化 剂最佳用量为 0.4 equiv.
表 5 酸催化剂对分子内 $C$-酰化反应的影响 ${ }^{a}$

Table 5 The effect of acid on intermolecular $C$-acylation

\begin{tabular}{clcc}
\hline Entry & Catalyst & Amount of catalyst/equiv. & Yield $^{b} / \%$ \\
\hline 1 & $98 \% \mathrm{H}_{2} \mathrm{SO}_{4}$ & 0.40 & 45 \\
2 & $\mathrm{PPA}$ & 0.40 & $<5$ \\
3 & $\mathrm{MsOH}$ & 0.40 & $<5$ \\
4 & $\mathrm{TsOH}$ & 0.40 & 80 \\
5 & $\mathrm{H}_{2} \mathrm{SO}_{4} @ \mathrm{SiO}_{2}$ & 0.40 & 90 \\
6 & $\mathrm{H}_{2} \mathrm{SO}_{4} @ \mathrm{SiO}_{2}$ & 0.30 & 80 \\
7 & $\mathrm{H}_{2} \mathrm{SO}_{4} @ \mathrm{SiO}_{2}$ & 0.35 & 85 \\
8 & $\mathrm{H}_{2} \mathrm{SO}_{4} @ \mathrm{SiO}_{2}$ & 0.45 & 82 \\
\hline
\end{tabular}

${ }^{a}$ Reaction conditions: compound $44 \mathrm{~g}(18.6 \mathrm{mmol}), 130{ }^{\circ} \mathrm{C}, 48 \mathrm{~h}$, dry toluene $(50 \mathrm{~mL}) .{ }^{b}$ Isolated yield.

1.3.3 硅胶负载硫酸催化分子内 $\mathrm{C}$-酰化反应机理的 探讨

根据表 5 所示结果，在化合物 $\mathbf{4}$ 的分子内 C-酰化反 应中, 硅胶负载硫酸的催化活性比浓硫酸还要高. 本课 题组曾报道在苯并氧杂葱的合成中, 硅胶包覆磁性纳米 粒子负载酸性离子液体的效果好于相应的均相酸性离 子液体，推测其原因是原料可以氢键等弱相互作用聚集 于硅胶表面，造成局部浓度增加，有利于反应速率增大; 同时酸性硅羟基可以以氢键形式活化原料，使得反应更 易进行 ${ }^{[22]}$. 因此, 我们推测化合物 4 在硅胶负载硫酸催 化下的分子内 $C$-酰化反应机理如 Scheme 3 所示. 首先 是化合物 4 中的酮羰基与硅胶表面的羟基形成氢键，这 个氢键可以促进䣶羰基的烯醇互变, 同时硫酸中的自由 氢质子对酯链中的乙氧基结合，脱去乙醇分子后生成酰 基正离子. 最后酰基正离子进攻烯醇中的双键完成酰化 反应.

\section{2 结论}

以 1,3-环已二酮为原料, 合成了一种新型双环多羰 基化合物双环[3.3.1]壬烷-2,6,9-三酮，并推测其反应机 


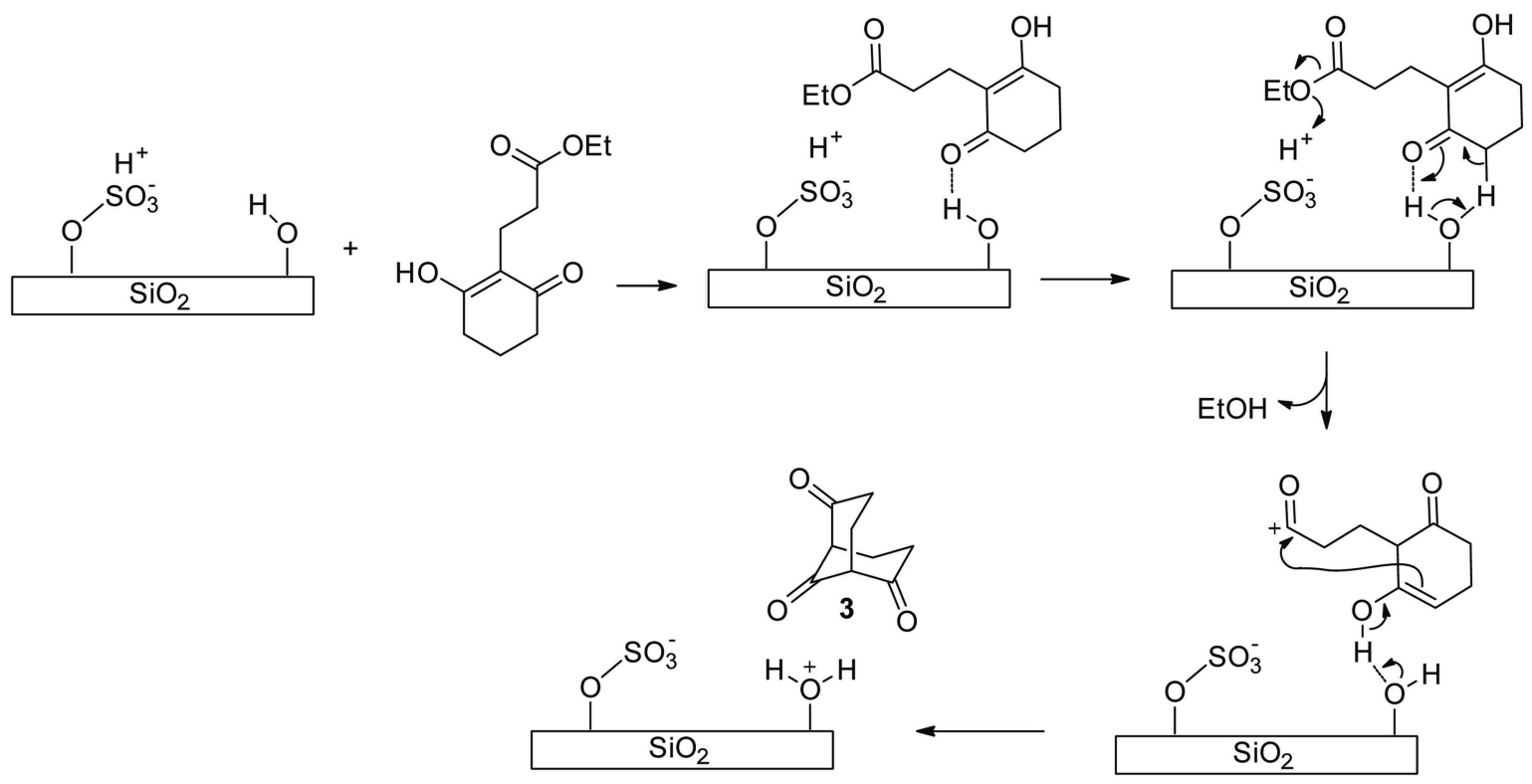

Scheme 3

理, 为双环[3.3.1]壬烷多酮骨架的构建提供了一种简单 有效的成环方法. 通过探讨三条路线, 得出最优方法. 其中第一条路线以 1,3-环已二酮和丙烯醛为原料经过 Michael 加成、分子内 Aldol 缩合和氧化三步得到产物, 总产率为 $43 \%$. 第二条路线以 1,3 -环己二酮和丙烯酸乙 酯为原料, 在吗啉存在经烯胺化、Michael 加成和碱催化 分子内 C-酰化三步反应 “一锅煮” 得到目标产物, 产率 为 $20 \%$. 第三条路线 1,3-环己二酮与丙烯酸乙酯为原料 经 Michael 加成和酸催化分子内 C-酰化反应两步生成目 标产物，总收率达到 $83 \%$. 本路线操作简便、产率高、 后处理方便、环境友好.

\section{3 实验部分}

\section{1 仪器与试剂}

Nicolet 傅里叶变换红外光谱仪, 美国 Thermofisher 公司; Bruker Avance-III DRX $500 \mathrm{MHz}$ 核磁共振仪, 德 国 Bruker 公司; Vario EL-III 型元素分析仪, 德国 Elemetar 公司; WRS-1B数字熔点仪, 上海申光仪器仪表 有限公司; 所有药品和试剂均为国产市售分析纯, 未经 处理直接使用, 无水溶剂按标准方法处理.

\section{2 实验步骤}

\subsubsection{6-羟基双环[3.3.1]壬烷-2,9-二酮(2)的合成}

氧化铝(7.65 g, $75 \mathrm{mmol})$ 在 $200{ }^{\circ} \mathrm{C}$ 下活化 $6 \mathrm{~h}$ 待用. 在研钵中加入 1,3-环已二酮(1.68 g, $15 \mathrm{mmol}$ )与活化的 氧化铝(7.65 g, $75 \mathrm{mmol})$, 室温下研磨 $10 \mathrm{~min}$, 在冰浴条 件下缓慢滴加丙烯醛 $(1.02 \mathrm{~mL}, 15 \mathrm{mmol})$, 滴加完成后, TLC 跟踪反应进程, $6 \mathrm{~h}$ 后反应结束. 加入二氯甲烷(100 $\mathrm{mL})$, 过滤, 用二氯甲烷洗涤, 浓缩, 硅胶柱层析 $[V($ 石
油醚)： $V$ (乙酸乙酯) $=1 ： 3$ ]得到化合物 $2(1.2 \mathrm{~g}, 48 \%)$. 白色固体，m.p. 67.9 68.2 ${ }^{\circ} \mathrm{C} ;{ }^{1} \mathrm{H}$ NMR $(500 \mathrm{MHz}$, $\left.\mathrm{CDCl}_{3}\right) \delta: 5.49(\mathrm{~m}, 1 \mathrm{H}), 4.12(\mathrm{~d}, J=4.7 \mathrm{~Hz}, 1 \mathrm{H}), 2.40 \sim$ $2.38(\mathrm{~m}, 4 \mathrm{H}), 2.34(\mathrm{t}, J=6.8 \mathrm{~Hz}, 2 \mathrm{H}), 2.02 \sim 1.87(\mathrm{~m}, 2 \mathrm{H})$, $1.82(\mathrm{~s}, 2 \mathrm{H}) ;{ }^{13} \mathrm{C} \mathrm{NMR}\left(126 \mathrm{MHz}, \mathrm{CDCl}_{3}\right) \delta:$ 198.67, 169.50, 111.59, 93.80, 36.55, 28.60, 26.99, 20.83, 14.23; IR (KBr) v: $3442,766(\mathrm{O}-\mathrm{H}), 2940,1452(\mathrm{C}-\mathrm{H}), 1683$, $(\mathrm{C}=\mathrm{O}), 1228(\mathrm{C}-\mathrm{O}) \mathrm{cm}^{-1}$. Anal. calcd for $\mathrm{C}_{9} \mathrm{H}_{12} \mathrm{O}_{3}: \mathrm{C}$ 63.30, H 7.08; found C 63.07, H 7.16.

3.2.2 3-(2-差基-6-氧代环己-1-烯-1-基)丙酸乙酯(4) 的合成

将 1,3-环己二酮 $(2.24 \mathrm{~g}, 20 \mathrm{mmol})$ 溶于 DMF (8 mL) 中, 冰浴下分批加入氢化钠 $(0.72 \mathrm{~g}, 30 \mathrm{mmol})$, 完全溶解 后, 常温下加入丙烯酸乙酯 $(2.13 \mathrm{~mL}, 20 \mathrm{mmol})$, 加热至 $80{ }^{\circ} \mathrm{C}$ 反应, TLC 跟踪反应进程, $4 \mathrm{~h}$ 后反应结束, 加入盐 酸 $(15 \mathrm{~mL}, 2 \mathrm{~mol} / \mathrm{L})$ 将溶液调至酸性, 将混合物转入水 (50 mL)中, 析出大量微白色固体, 抽滤, 在 $80{ }^{\circ} \mathrm{C}$ 烘箱 中干燥 $10 \mathrm{~h}$, 得到化合物 4 (4 g, 92\%). 乳白色固体, m.p. $125.0 \sim 126.2{ }^{\circ} \mathrm{C} ;{ }^{1} \mathrm{H}$ NMR $\left(500 \mathrm{MHz}, \mathrm{CDCl}_{3}\right) \delta: 9.58(\mathrm{~s}$, $1 \mathrm{H}), 4.16(\mathrm{q}, J=7.0 \mathrm{~Hz}, 2 \mathrm{H}), 2.51 \sim 2.46(\mathrm{~m}, 4 \mathrm{H}), 2.45(\mathrm{t}$, $J=6.5 \mathrm{~Hz}, 2 \mathrm{H}), 2.31(\mathrm{t}, J=6.5 \mathrm{~Hz}, 2 \mathrm{H}), 1.90(\mathrm{p}, J=6.5$ $\mathrm{Hz}, 2 \mathrm{H}), 1.25$ (t, $J=6.5 \mathrm{~Hz}, 3 \mathrm{H}) ;{ }^{13} \mathrm{C} \mathrm{NMR}(126 \mathrm{MHz}$, $\left.\mathrm{CDCl}_{3}\right) \delta: 198.32,177.52,173.08,114.08,61.13,36.08$, $32.65,28.94,19.95,16.18,13.52$; IR (KBr) v: 3450, 722 $(\mathrm{O}-\mathrm{H}), 3060,903(\mathrm{C}=\mathrm{C}), 1768(\mathrm{C}=\mathrm{O}), 2940,1452$ $(\mathrm{C}-\mathrm{H}), 1230(\mathrm{C}-\mathrm{O}) \mathrm{cm}^{-1}$. Anal. calcd for $\mathrm{C}_{11} \mathrm{H}_{16} \mathrm{O}_{4}: \mathrm{C}$ 62.25, H 7.60; found C 62.08, H 7.49. 


\subsection{3 双环[3.3.1]壬烷-2,6,9-三酮(化合物 3)的合成}

路线 1: 在研针中加入过硫酸氢钾 $(2.35 \mathrm{~g}, 3.8$ $\mathrm{mmol})$, 室温下研磨 $10 \mathrm{~min}$, 加入乙酸乙酯 $(8 \mathrm{~mL})$ 常温 搅拌 $2 \mathrm{~h}$. 先后加入邻碘苯甲酸 $(0.09 \mathrm{~g}, 0.38 \mathrm{mmol})$ 、化合 物 $2(0.8 \mathrm{~g}, 4.76 \mathrm{mmol})$ 的乙酸乙酯 $(5 \mathrm{~mL})$ 溶液, 加热回 流反应, TLC 跟踪反应进程, $8 \mathrm{~h}$ 后反应结束, 过滤, 乙 酸乙酯洗涤, 浓缩, 硅胶柱层析 $[V($ 乙酸乙酯 $): V($ 石油 醚 $)=1 ： 3$ ]得到化合物 $3(0.7 \mathrm{~g}, 90 \%)$. 白色固体, m.p. $144.2 \sim 145.0{ }^{\circ} \mathrm{C} ;{ }^{1} \mathrm{H}$ NMR $\left(500 \mathrm{MHz}, \mathrm{CDCl}_{3}\right) \delta: 2.72 \sim$ $2.67(\mathrm{~m}, 1 \mathrm{H}), 2.64 \sim 2.55(\mathrm{~m}, 2 \mathrm{H}), 2.47 \sim 2.44(\mathrm{~m}, 1 \mathrm{H})$, $2.13 \sim 2.06(\mathrm{~m}, 1 \mathrm{H}) ;{ }^{13} \mathrm{C} \mathrm{NMR}\left(126 \mathrm{MHz}, \mathrm{CDCl}_{3}\right) \delta$ : $198.67,169.50,111.59,93.80,36.55,28.60,26.99,20.83$, 14.23; IR (KBr) v: 1770, $1644(\mathrm{C}=\mathrm{O}), 2950,1438,1354$ $(\mathrm{C}-\mathrm{H}) \mathrm{cm}^{-1}$. Anal. calcd for $\mathrm{C}_{9} \mathrm{H}_{10} \mathrm{O}_{3}$ : C 65.05, $\mathrm{H}$ 6.07; found $\mathrm{C}$ 64.82, $\mathrm{H}$ 5.92; HRMS calcd for $\mathrm{C}_{9} \mathrm{H}_{10} \mathrm{O}_{3}(\mathrm{M}+\mathrm{H})^{+}$ 166.9603, found 167.0630 .

路线 2: 将 1,3-环己二酮 $(2.24 \mathrm{~g}, 20 \mathrm{mmol})$ 、吗啉 (5.23 mL, $60 \mathrm{mmol})$ 加入到无水甲苯 $(50 \mathrm{~mL})$ 中, 先 100 ${ }^{\circ} \mathrm{C}$ 反应 $1 \mathrm{~h}$, 然后升温至 $130{ }^{\circ} \mathrm{C}$ 反应. TLC 跟踪反应进 程, $5 \mathrm{~h}$ 反应结束, 低压除去吗啉与甲苯后, 加入丙烯酸 乙酯 $(2.13 \mathrm{~mL}, 20 \mathrm{mmol}) 、$ 二氯甲烷 $(30 \mathrm{~mL})$ 、烘干待用 的 4A 分子笁, 加热回流反应. TLC 跟踪反应进程, $8 \mathrm{~h}$ 后 反应结束, 过滤, 用二氯甲烷洗涤, 浓缩, 硅胶柱层析 $[V($ 乙酸乙酯 $): V($ 石油醚 $)=1: 5 \sim 1: 3$ ]得到化合物 3 (0.66 g, 20\%).

路线 3: 将硅胶 $(60 \sim 80$ 目, $20.08 \mathrm{~g})$ 加入到硫酸(10 $\mathrm{mL}, 98 \%)$ 与二氯甲烷 $(50 \mathrm{~mL})$ 中, 加热回流反应, $4 \mathrm{~h}$ 后 反应结束, 浓缩, $500{ }^{\circ} \mathrm{C}$ 炦烧 $4 \mathrm{~h}$, 得到白色流沙状硅胶 负载浓硫酸 $(24 \mathrm{~g})$. 将化合物 $4(4 \mathrm{~g}, 18.6 \mathrm{mmol})$ 、硅胶负 载浓硫酸 $(1.17 \mathrm{~g})$ 、少量已烘好待用的 $4 \AA$ 分子笁加入到 无水甲苯 $(30 \mathrm{~mL})$ 中, 先 $100{ }^{\circ} \mathrm{C}$ 反应 $1 \mathrm{~h}$, 后升温至 130 ${ }^{\circ} \mathrm{C}$ 反应, TLC 跟踪反应进程, $48 \mathrm{~h}$ 后反应结束. 过滤, 用
二氯甲烷 $(200 \mathrm{~mL})$ 洗涤滤饼, 浓缩, 硅胶柱层析 $[V($ 乙酸 乙酯)： $V$ (石油醚) $=1 ： 3$ ]得到化合物 $3(2.81 \mathrm{~g}, 90 \%)$.

\section{References}

[1] Marvell, E. N.; Knutson, R. S.; McEwen, T.; Sturmer, D.; Federici, W.; Salisbury, K. J. Org. Chem. 1970, 35, 392.

[2] Tang, J. M.; Guo, J. W.; Liu, S. Adv. Fine Petrochem. 2003, (4), 46 (in Chinese).

(谭镜名, 郭建维, 刘州, 精细石油化工进展, 2003, (4), 46.)

[3] Pouplin, T.; Tolon, B.; Nuhant, P.; Delpech, B.; Marazano, C. Eur. J. Org. Chem. 2007, 5117.

[4] Tori, M.; Hisazumi, K.; Wada, T.; Sono, M.; Nakashima K. Tetrahedron: Asymmetry 1999, 10, 961.

[5] Pradhan, T. K.; Hassner, A. Synlett 2007, 1071.

[6] Danishefsky, S.; Migdalof, B. H. Tetrahedron 1969, 50, 4331.

[7] Zhang, Q.; Porco, Jr. J. A. Org. Lett. 2012, 14, 1796.

[8] Mehta, G.; Bera, M. K.; Chatterjee, S. Tetrahedron Lett. 2008, 49, 1121.

[9] Mehta, G.; Das, M.; Kundu, U. K. Tetrahedron Lett. 2012, 53, 4538.

[10] Noël, R.; Vanucci-Bacqué, C.; Fargeau-Bellassoued, M.-C.; Lhommet, G. J Org Chem. 2005, 22, 9044.

[11] Ranu, B. C.; Bhar, S. Tetrahedron 1992, 48, 1327.

[12] Ranu, B. C.; Bhar, S. Sarkar, D. C. Tetrahedron Lett. 1991, 32, 2811.

[13] Filippini, M.-H.; Faure, R.; Rodriguez, J. J. Org. Chem. 1995, 60, 6872.

[14] Lyttle, M. H.; Streitwieser, A.; Miller, M. J. J. Org. Chem. 1989, 54, 2331.

[15] Hawkins, R. T.; Hsu, R. S.; Wood, S. G. J. Org. Chem. 1978, 43, 4648.

[16] Dondoni, A.; Perrone, D. Org. Synth. 2004, 10, 320.

[17] Takahashi, H.; Kajimoto, T.; Tsuji, J. Synth. Commun. 1972, 2, 181.

[18] Hu, Z. M.; Du, H. X.; Leung, C. F.; Liang, H. J.; Lau, T. C. Ind. Eng. Chem. Res. 2011, 50, 12288.

[19] Uyanik, M.; Akakura, M.; Ishihara, K. J. Am. Chem. Soc. 2009, $131,251$.

[20] Boltukhina, E. V.; Sheshenev, A. E.; Lyapkalo, I. M. Tetrahedron 2011, 67, 5382.

[21] Mitoshi, K.; Takahiko, N.; Shigeru, S.; Katsuhiro, I.; Hisao, N.; Nobuyuki, H. Synlett 1997, 1472.

[22] Zhang, Q.; Su, H.; Luo, J.; Wei, Y. Y. Green Chem. 2012, 14, 201.

(Li, L.; Fan, Y.) 